\title{
DIATOMÁCEAS PERIFÍTICAS DOS ARROIOS SAMPAIO E SAMPAINHO, RIO GRANDE DO SUL, BRASIL
}

\author{
Maria Angélica Oliveira ${ }^{1}$ \\ Lezilda Carvalho Torgan ${ }^{2}$ \\ Silvana Corrêa Rodrigues ${ }^{2}$
}

Recebido em 20/02/2001. Aceito em 10/09/2001.

RESUMO - (Diatomáceas perifíticas dos arroios Sampaio e Sampainho, Rio Grande do Sul, Brasil). Estudo taxonômico de diatomáceas perifíticas em substrato artificial (fio de poliamida) foi realizado nos arroios Sampaio e Sampainho, localizados na Depressão Central do Estado do Rio Grande do Sul. A investigação baseou-se em amostragens mensais, realizadas no período de um ano (julho de 1994 a junho de 1995). Foram identificados, descritos e ilustrados vinte e um táxons infragenéricos, distribuídos em treze gêneros e onze famílias. Achnanthes rupestoides Hohn, Diadesmis brekkaensis (Petersen) D. G. Mann, Navicula tenelloides Hustedt e Placoneis elginensis (Gregory) E. J. Cox são registrados pela primeira vez em ambientes lóticos no Estado.

Pavavras-chave - Bacillariophyta, taxonomia, arroios, sul do Brasil

\begin{abstract}
Periphitic diatoms from Sampaio and Sampainho streams, Rio Grande do Sul, Brazil). Taxonomic study of periphitic diatoms on an artificial substrate (polyamide threads) was undertaken in the Sampaio and Sampainho streams, that are located in the Depressão Central region of Rio Grande do Sul State. The investigation was based on monthly sampling taken from July 1994 to June 1995. Twenty one infrageneric taxa distributed in thirteen genera and eleven families were identified, described and illustrated. Achnanthes rupestoides Hohn, Diadesmis brekkaensis (Petersen) D. G. Mann, Navicula tenelloides Hustedt and Placoneis elginensis (Gregory) E. J. Cox are registered for the first time in lotic environments in this state.
\end{abstract}

Key words - Bacillariophyta, taxonomy, stream, south of Brazil

\section{Introdução}

O arroio Sampaio localiza-se na Depressão Central do estado do Rio Grande do Sul $\left(29^{\circ} 21^{\prime}-29^{\circ} 33^{\prime} \mathrm{S}\right.$ e $\left.51^{\circ} 58^{\prime}-52^{\circ} 22^{\prime} \mathrm{W}\right)$. Como integrante da bacia do rio Taquari, este arroio nasce no alto da Serra Geral e drena suas águas em região de alto relevo e declividade. Ao longo de seu percurso de $64,4 \mathrm{~km}$, recebe a contri- buição de vários arroios de menor porte, entre eles, o arroio Sampainho.

O conhecimento da comunidade perifítica dos arroios Sampaio e Sampainho teve início com o estudo limnológico de Oliveira (Oliveira, 1996 e Oliveira \& Schwarzbold, 1998). Com base nesse estudo, constatou-se a ocorrência de trechos impactados pela atividade agro-pastoril do entorno, evidenciando-se os efeitos da ocu-

\footnotetext{
Bolsista de doutorado da Fundação Coordenação de Aperfeiçoamento de Pessoal de Nível Superior

2 Museu de Ciências Naturais da Fundação Zoobotânica do Rio Grande do Sul, Porto Alegre, Caixa Postal 1188, CEP 90690-000, RS, Brasil. e-mail: torgan@cpovo.net
} 
pação humana na qualidade das águas da microbacia.

As diatomáceas destacaram-se no perifíton e, portanto, foram objetos do estudo posteriormente realizado sobre as associações de espécies características dos diferentes trechos do arroio Sampaio (Oliveira et al., 2001). Com a continuidade da investigação taxonômica de diatomáceas nos arroios Sampaio e Sampainho, foram observadas espécies de ocorrência rara, que não tinham sido ainda registradas nestes ambientes. Este fato motivou a realização deste trabalho, que tem por objetivo caracterizar e ilustrar espécies de diatomáceas que compõem a comunidade perifítica nestes arroios, ampliando desta forma o conhecimento da diversidade de microalgas em ambientes lóticos do Estado.

\section{Material e métodos}

O estudo baseou-se na análise de 25 amostras coletadas no período de julho de 1994 a junho de 1995, nos trechos superior (estação 6) e médio (estações 1 e 3) do arroio Sampaio e no trecho inferior (estação 2) do arroio Sampainho. O material perifítico foi obtido de substrato artificial (fios de poliamida) exposto por trinta dias na subsuperfície da água, sendo preservado posteriormente em solução F.A.A. (Gough \& Woelkerling, 1976). O material foi oxidado, segundo a técnica de procedimento rápido descrita em Müller-Melchers \& Ferrando (1956) e a identificação das diatomáceas foi efetuada em lâminas permanentes montadas com Nafrax (I.R. 1,74).

As amostras e lâminas mencionadas no texto encontram-se depositadas e registradas no Herbário Prof. Dr. Alarich R.H. Schultz (HAS), do Museu de Ciências Naturais da Fundação Zoobotânica do Rio Grande do Sul. Utilizou-se o sistema de classificação de Round et. al. (1990) para o enquadramento dos táxons. A identificação taxonômica baseou-se nos trabalhos de Van Heurck (1885), Hustedt (1930;
1937-8; 1961), Cleve-Euler (1953), Hohn \& Hellerman (1963), Patrick \& Reimer (1966; 1975), Rivera (1974), Camburn et. al. (1978), Schoeman \& Archibald (1978, 1979), Germain (1981), Krammer \& Lange-Bertalot (1986), Jahn (1986), Williams \& Round (1987), Cox (1987), Round et al. (1990), Krammer \& Lange-Bertalot (1991a; 1991b), Hürlimann \& Straub (1991) e Hartley (1996).

\section{Resultados}

Os vinte e um táxons infragenéricos identificados estão distribuídos em treze gêneros e onze famílias, como se segue:

Divisão Bacillariophyta

Classe Fragilariophyceae F. E. Round 1990

Subclasse Fragilariophycidae F. E. Round 1990 Ordem Fragilariales Silva 1962 sensu emend. Família Fragilariaceae Grev. 1833

Fragilaria H. C. Lyngb. 1819

1. Fragilaria capucina Desm., Plantes Cryptogames de la France. 10(453). 1825.

Fig. 1

Valvas linear-lanceoladas com região central levemente dilatada em uma das margens. Extremidades ligeiramente rostradas. Área central presente. Comprimento $27 \mu \mathrm{m}$; largura 5,4 $\mu \mathrm{m}$; estrias transapicais robustas e pararelas, $11 \mathrm{em}$ $10 \mu \mathrm{m}$.

Material examinado: BRASIL. Rio Grande do Sul: Mato Leitão, Arroio Sampaio, estação 1, V/1995, lâmina n. 4899 (HAS 34015).

2. Fragilaria radians (Kütz.) Williams \& Round, Diatom Res. 2: 269. 1987.

Synedra radians Kütz., Bacillariophyceae: 64, est. 14 , f. 7.1844.

Fig. 2

Valvas linear-lanceoladas. Região central levemente dilatada em ambas as margens. Extremidades rostrada-arredondadas. Comprimento 
$54,6 \mu \mathrm{m}$; largura $3 \mu \mathrm{m}$; estrias transapicais paralelas, 16 em $10 \mu \mathrm{m}$.

Material examinado: BRASIL. Rio Grande do

Sul: Mato Leitão, Arroio Sampaio, estação 1, VII/1994, lâmina n. 4893 (HAS 34009).

Classe Bacillariophyceae Haeckel 1878 emend D. G. Mann

Subclasse Eunotiophycidae D. G. Mann 1990

Ordem Eunotiales Silva 1962

Família Eunotiaceae Kütz. 1844

Eunotia C. G. Ehr. 1837

3. Eunotia curvata (Kütz.) Lagerst. var. curvata, K. Svenska Vet.-Akad. Handl. 41: 61. 1884. Fig. 3

Valvas arqueadas estreitando-se levemente em direção às extremidades. Margem dorsal convexa, margem ventral levemente côncava. Extremidades arredondadas com nódulos terminais pequenos. Comprimento $63 \mu \mathrm{m}$; largura 3,6 $\mathrm{mm}$; estrias transapicais paralelas, $16 \mathrm{em} 10 \mu \mathrm{m}$.

Material examinado: BRASIL. Rio Grande do Sul: Mato Leitão, Arroio Sampaio, estação 1, VII/1994, lâmina n. 4892 (HAS 34009).

4. Eunotia praerupta Ehr. var. bidens (Ehr.) Grun. in Cleve \& Grun., K. Svenska Vet. - Akad. Handl. 17: 109. 1880.

Fig. 4

Valvas com duas ondulações na margem dorsal e margem ventral levemente côncava. Extremidades largamente truncadas. Comprimento $46,8 \mu \mathrm{m}$; largura 9,6 $\mu \mathrm{m}$; estrias transapicais paralelas, 11 em $10 \mu \mathrm{m}$.

Material examinado: BRASIL. Rio Grande do Sul, Mato Leitão, Arroio Sampainho, estação 2, VIII/1994, lâmina n.4903 (HAS 34017).

Subclasse Bacillariophycidae D. G. Mann 1990 Ordem Cymbellales D. G. Mann 1990

Família Cymbellaceae Grev. 1833

Cymbella C. Ag. 1830
5. Cymbella affinis Kütz., Die Kieselschaligen Bacillarien oder Diatomeen: 80, f. 6-15. 1844. Fig. 5

Valvas com margens dorsal e ventral convexas. Extremidades sub-rostradas arredondadas, ventralmente projetadas. Rafe filiforme excêntrica com fissuras terminais dorsalmente fletidas. Comprimento $37,5-39 \mu \mathrm{m}$; largura $10-11 \mu \mathrm{m}$; estrias transapicais levemente radiadas, 9-10 em $10 \mu \mathrm{m}$.

Material examinado: BRASIL. Rio Grande do Sul: Mato Leitão, Arroio Sampainho, estação 2, IX/1994, lâmina n. 4904 (HAS 34018); Arroio Sampainho, estação 2, XI/1994, lâmina n. 4906 (HAS 34020).

Encyonema F. T. Kütz. 1833

6. Encyonema perpusillum (A. Cleve) D. G. Mann in Round, Crawford \& Mann, The Diatoms. Biology and Morphology of the Genera: 666, 1990.

Cymbella perpusilla A. Cleve, K. Svenska Vet.Akad. Handl. 27: 19. 1895.

Fig. 6

Valvas com margens dorsal e ventral convexas. Extremidades sub-rostradas arredondadas, ventralmente projetadas. Rafe filiforme excêntrica com fissuras terminais ventralmente fletidas e, extremidades proximais dorsalmente fletidas. Comprimento $21 \mu \mathrm{m}$; largura 5,4 $\mu \mathrm{m}$; estrias transapicais robustas, levemente radiadas, $10 \mathrm{em}$ $10 \mu \mathrm{m}$ no centro e $16 \mathrm{em} 10 \mu \mathrm{m}$ nas extremidades valvares.

Material examinado: BRASIL. Rio Grande do Sul: Mato Leitão, Arroio Sampaio, estação 6, VI/ 1995, lâmina n. 4929 (HAS 34070).

Placoneis C. Mereschk. 1903

7. Placoneis clementis (Grun.) E. J. Cox, Diatom Res. 2(2): 155. 1987.

Navicula clementis Grun., Beitr. Palaeont. Oesterr. 2:144, est. 30, f. 52. 1882.

Fig. 7 


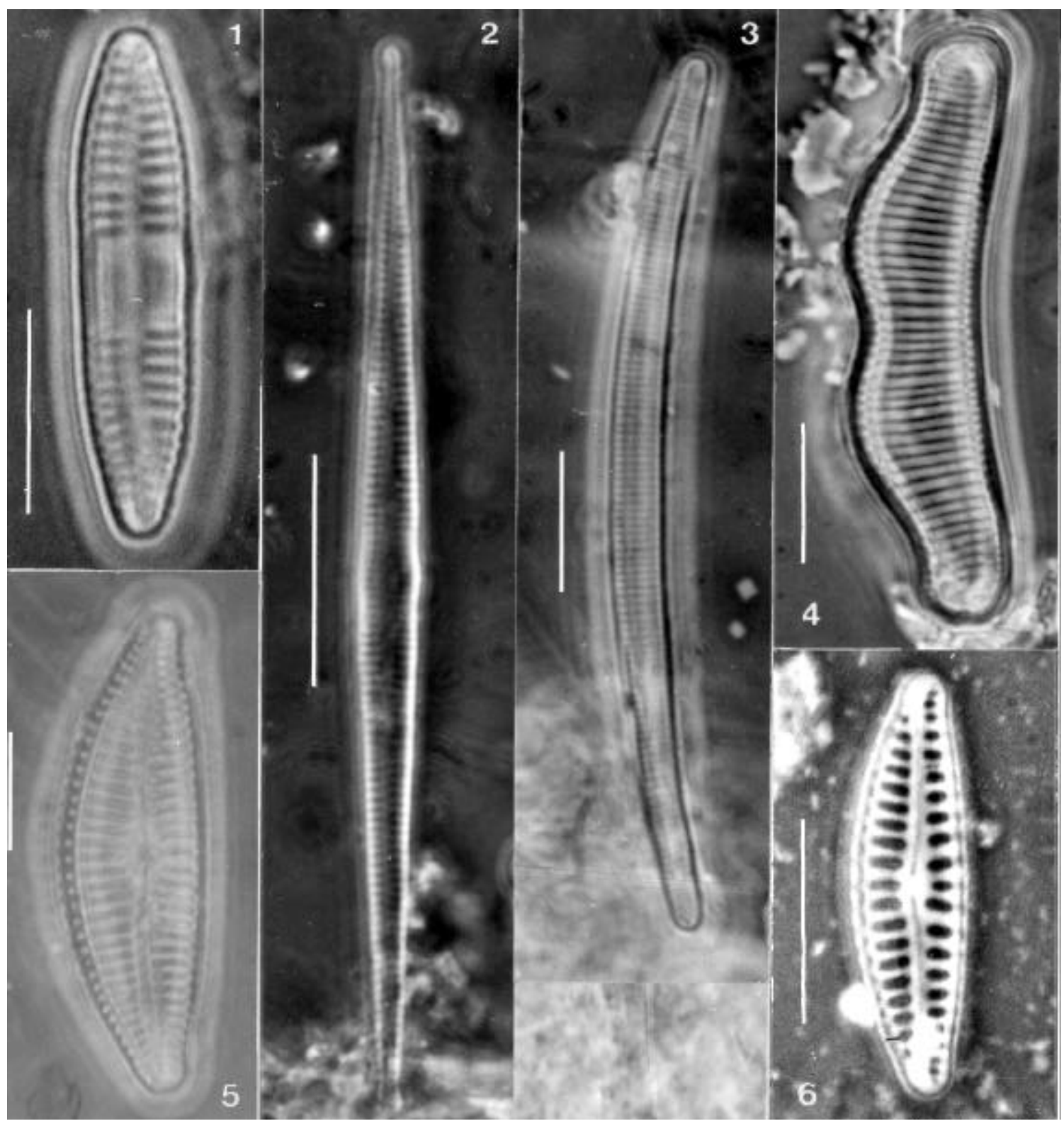

Figuras 1-6. 1. Fragilaria capucina; 2. Fragilaria radians; 3. Eunotia curvata var. curvata; 4. Eunotia praerupta var. bidens; 5. Cymbella affinis; 6. Encyonema perpusillum.

Valvas elíptica-lanceoladas com extremidades levemente rostradas. Rafe linear reta com extremidades proximais dilatadas em grandes nódulos. Área central irregular com presença de um a três poros isolados. Comprimento $39-51 \mu \mathrm{m}$; largura $14,5-16 \mu \mathrm{m}$, estrias transapicais radiadas com pontuações nítidas, 13-14 em 10 $\mu \mathrm{m}$.
Material examinado: BRASIL. Rio Grande do Sul: Mato Leitão, Arroio Sampaio, estação 1, XII/1994, lâmina n. 4895 (HAS 34013); Arroio Sampaio, estação 3, III/1995, lâmina n. 4913 (HAS 34033).

Comentários: foram observados indivíduos com um ou três poros isolados na área central, sendo 
que Patrick \& Reimer (1966) mencionam a presença de dois poros.

8. Placoneis elginensis (Greg.) E. J. Cox, Diatom Res. 2(2): 155, f. 20-27, 34. 1987.

Navicula elginensis (Greg.) Ralfs in Pritchard, Hist. Infusoria 902. 1861.

Fig. 8

Valvas elípticas com extremidades capitada-rostradas. Rafe linear reta com extremidades distais curvadas. Área central retangular com estrias irregulares e curtas. Comprimento $22,2 \mu \mathrm{m}$; largura $7 \mu \mathrm{m}$; estrias transapicais radiadas, 10 $12 \mathrm{em} 10 \mu \mathrm{m}$.

Material examinado: BRASIL. Rio Grande do Sul: Mato Leitão, Arroio Sampaio, estação 6, VI/1994, lâmina n. 4926 (HAS 34059).

Comentários: primeira citação para o estado do Rio Grande do Sul.

Família Gomphonemataceae Kütz. 1844

Gomphonema C. G. Ehr. 1832

9. Gomphonema augur Ehr., Ber. Akad. Wiss. Berlin: 211. 1940.

Fig. 9

Valvas heteropolares clavadas, dilatada na metade superior. Extremidades capitada-rostradas. Rafe linear reta. Área central unilateral, formada pelo encurtamento de uma estria mediana. Comprimento $40-41 \mu \mathrm{m}$; largura $12-13 \mu \mathrm{m}$; estrias transapicais paralelas, pontuadas, radiadas nas extremidades 10-12 em 10 $\mu \mathrm{m}$.

Material examinado: BRASIL. Rio Grande do Sul: Mato Leitão, Arroio Sampaio, estação 3, VIII/1994, lâmina n. 4911 (HAS 34028).

Ordem Achnanthales Silva 1862

Família Achnanthaceae Küt. 1844 sensu emend. Achnanthes Bory de St.-Vincent 1822

10. Achnanthes coarctata (Bréb.) Grun. in Cleve \& Grun., K. Svenska Vet.-Akad. Handl. 17(2): 20. 1880.
Fig. 10

Valvas linear-elípticas, constricta na região central. Rafe linear reta com extremidades proximais dilatadas em nódulo. Área central retangular sem estrias. Comprimento $30 \mu \mathrm{m}$, largura $7,2 \mu \mathrm{m}$; estrias transapicais radiadas, nitidamente pontuadas, $16 \mathrm{em} 10 \mu \mathrm{m}$.

Material examinado: BRASIL. Rio Grande do Sul: Mato Leitão, Arroio Sampainho, estação 2, VI/1994, lâmina n. 4901(HAS 34016).

11. Achnanthes rupestoides Hohn, Trans. Am. Microsc. Soc. 80: 154, est. 1, f. 3-4. 1861.

Fig. 11-12

Valvas elípticas. Rafe linear reta com extremidades proximais dilatadas em nódulo. Área central retangular com estrias curtas. Valva sem rafe com área axial lanceolada, sem área central. Comprimento $11,4-13,2 \mu \mathrm{m}$, largura 5,4-7,2 $\mu \mathrm{m}$; estrias transapicais radiadas, $18-20 \mathrm{em} 10 \mu \mathrm{m}$.

Material examinado: BRASIL. Rio Grande do Sul: Mato Leitão, Arroio Sampaio, estação 1, VI/1994, lâmina n. 4892 (HAS 34009); Arroio Sampaio, estação 6, VI/1994, lâmina n. 4929 (HAS 34070).

Comentários: a transferência da espécie $A$. rupestoides Hohn ao novo gênero Psammonthidium Bukhtiyarova \& Round, depende de estudos ao microscópio eletrônico, sendo, portanto, mantida a denominação original, segundo Krammer \& Lange-Bertalot (1991b). Primeira citação para o estado do Rio Grande do Sul.

Ordem Naviculales Bessey 1907

Subordem Neidiineae D. G. Mann 1990

Família Diadesmidaceae D. G. Mann 1990

Diadesmis F. T. Kütz. 1844

12. Diadesmis brekkaensis (J. B. Petersen) D. G. Mann in Round, Crawford \& Mann, The Diatoms. Biology and Morphology of the Genera: 666. 1990. 
Navicula brekkaensis J. B. Petersen, Bot. Iceland. 2: 389. 1928.

Fig. 13

Valvas lineares levemente alargadas na região mediana. Extremidades largamente arredondadas. Rafe linear reta. Área central circular. Comprimento $16,8 \mu \mathrm{m}$; largura $2,9 \mu \mathrm{m}$; estrias transapicais delicadas, inconspícuas.

Comentários: segundo Krammer \& Lange-Bertalot (1986) esta espécie pode apresentar de 2436 estrias em $10 \mu \mathrm{m}$. Primeira citação para o estado do Rio Grande do Sul.

Material examinado: BRASIL. Rio Grande do Sul: Mato Leitão, Arroio Sampainho, estação 2, VI/1994, lâmina n. 4903(HAS 34017).

13. Diadesmis contenta (Grun. in V. H.) D. G. Mann in Round, Crawford \& Mann, The Diatoms. Biology and Morphology of the Genera: 666. 1990.

Navicula contenta Grun. ex. V. H., Synopsis des Diatomées de Belgique: 109. 1885.

Fig. 14

Valvas lineares levemente constrictas na região mediana. Extremidades largamente arredondadas. Rafe linear reta. Área central retangular. Comprimento $10,2 \mu \mathrm{m}$; largura $2,6 \mu \mathrm{m}$; estrias transapicais delicadas, inconspícuas.

Material examinado: BRASIL. Rio Grande do

Sul; Mato Leitão, Arroio Sampaio, estação 6, VI/1994, lâmina n. 4926 (HAS 34059).

Comentários: segundo Krammer \& Lange-Bertalot (1986), esta espécie pode apresentar de 2540 estrias em $10 \mu \mathrm{m}$.

Subordem Sellaphorineae D. G. Mann 1990 Família Sellaphoraceae C. Mereschk. 1902 Fallacia A. J. Stickle \& D. G. Mann 1990

14. Fallacia pygmaea (Kütz.) Stickle \& Mann in Round, Crawford \& Mann, The Diatoms. Biology and Morphology of the Genera: $554 \mathrm{e}$ 668. 1990.
Navicula pygmaea Kütz., Species Algarum: 77. 1849.

Fig. 15

Valvas elípticas com linhas hialinas longitudinais, em forma de lira, próximas à rafe. Rafe linear reta com extremidades proximais dilatadas em nódulo. Comprimento $26 \mu \mathrm{m}$; largura $9,6 \mu \mathrm{m}$; estrias transapicais finas, radiadas, 26 em $10 \mu \mathrm{m}$.

Material examinado: BRASIL. Rio Grande do Sul: Mato Leitão, Arroio Sampainho, estação 2, X/1994, lâmina n. 4905 (HAS 34019).

Sellaphora C. Mereschk. 1902

15. Sellaphora pupula (Kütz.) Mereschk., Ann. Mag. Nat. Hist. 9(7): 187, est. 4, f. 1-15. 1902. Navicula pupula Kütz. f. capitata (Hust.) Hust., Abh. Naturw. Ver. Bremen. 34(3): 282. 1957.

Fig. 16

Valvas lineares. Extremidades sub-capitadas. Rafe linear reta com extremidades proximais dilatadas em pequeno nódulo. Área central retangular, com estrias de tamanho irregular. Comprimento $44 \mu \mathrm{m}$; largura $10,2 \mu \mathrm{m}$; estrias transapicais finas, levemente radiadas, $16 \mathrm{em} 10 \mu \mathrm{m}$. Material examinado: BRASIL. Rio Grande do Sul: Mato Leitão, Arroio Sampaio, estação 1, XII/1994, lâmina n. 4896 (HAS 34013).

Família Pinnulariaceae D. G. Mann 1990

Pinnularia C.G. Ehr. 1843

16. Pinnularia borealis Ehr. var. rectangularis Carlson, Wiss. Ergebn. Schwedische Sudpolar Exped: 31, est. 3, f. 15. 1913.

Fig. 17

Valvas retangulares, dilatadas levemente na região central. Rafe linear reta com extremidades proximais dilatadas em grandes nódulos. Área central retangular com estrias mais curtas. Comprimento $44 \mu \mathrm{m}$; largura $10 \mu \mathrm{m}$; estrias transapicais, levemente radiadas, paralelas em direção às extremidades, $4-5 \mathrm{em} 10 \mu \mathrm{m}$. 


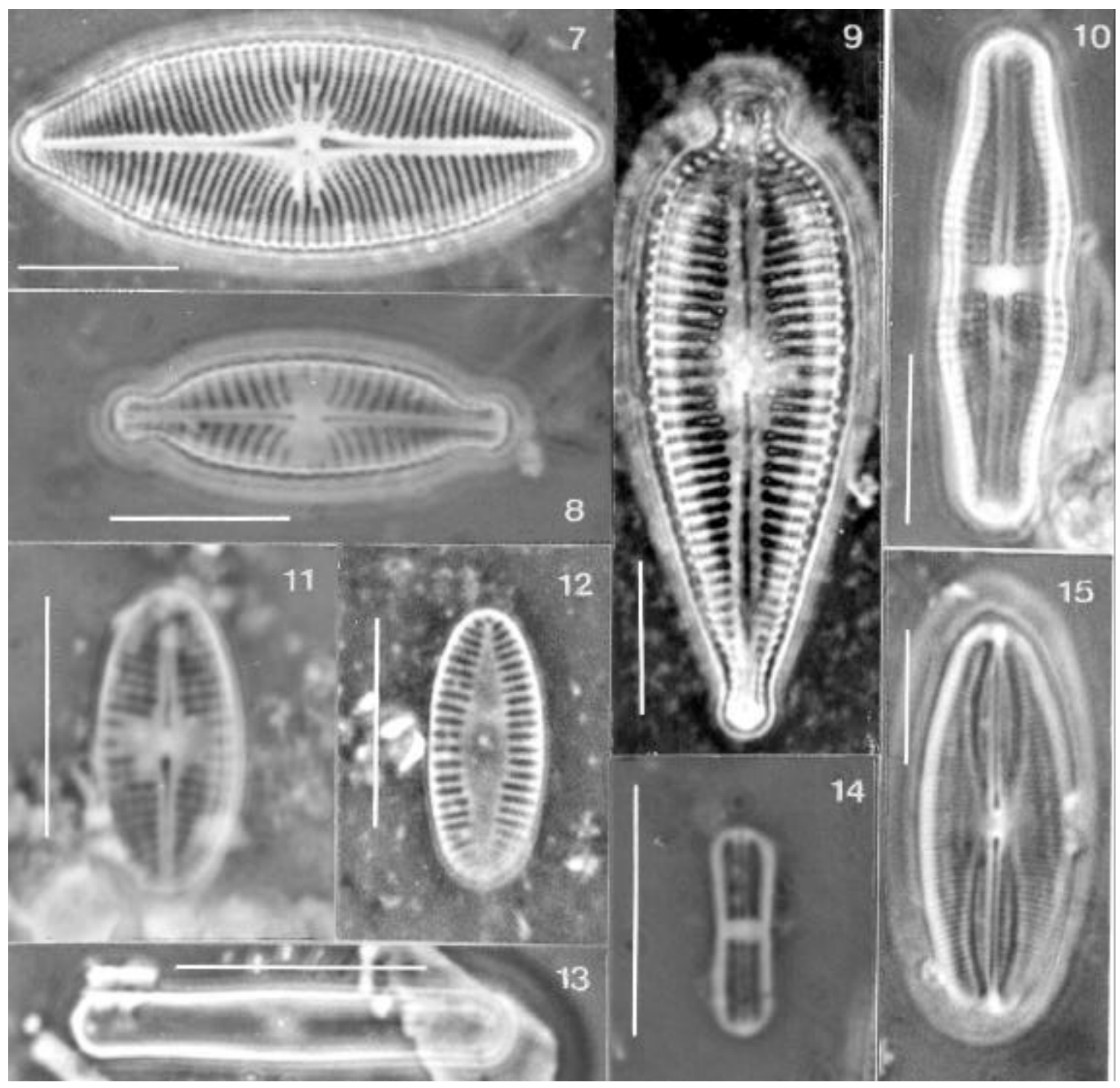

Figuras 7-15. 7. Placoneis clementis; 8. Placoneis elginensis; 9. Gomphonema augur; 10. Achnanthes coarctata; 11. Achnanthes rupestoides (valva com rafe); 12. Achnanthes rupestoides (valva sem rafe); 13. Diadesmis brekkaensis; 14. Diadesmis contenta; 15. Fallacia pygmaea.

Material examinado: BRASIL. Rio Grande do Sul: Mato Leitão, Arroio Sampaio, estação 1, XII/1994, lâmina n. 4896 (HAS 34013).

17. Pinnularia mesolepta (Ehr.) W. Sm., Synopsis of British Diatomaceae 1: 58, est. 19, f. 182. 1853.

Fig. 18
Valvas lineares com margens dorsais trionduladas, sendo a ondulação central bem menor em relação as outras duas. Extremidades largamente capitadas. Rafe linear reta com extremidades proximais fletidas para o lado. Área axial lanceolada, área central transversa. Comprimento 44,4-49,5 $\mu \mathrm{m}$; largura 7,8-9,0 $\mu \mathrm{m}$; estrias transapicais, radiadas na região central e convergentes nas extremidades, 9-10 em 10 $\mu \mathrm{m}$. 
Material examinado: BRASIL. Rio Grande do Sul: Mato Leitão, Arroio Sampaio, estação 1, IX/1994, lâmina n. 4894 (HAS 34011); Arroio Sampaio, estação 1, V/1995, lâmina n. 4899 (HAS 34015).

18. Pinnularia microstauron (Ehr.) Cleve, The Diatoms of Finland, Acta Soc. Fauna Flora fenn. 8(2): 28. 1891.

Fig. 19

Valvas lineares com extremidades levemente capitada-rostradas. Rafe linear reta com extremidades proximais fletidas para o lado. Área axial lanceolada, área central transversa. Compri-

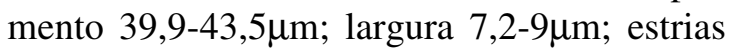
transapicais, radiadas na região central e convergentes nas extremidades, 9-10 em 10 $\mu \mathrm{m}$. Material examinado: BRASIL. Rio Grande do Sul: Mato Leitão, Arroio Sampaio, estação 1, lâmina n. 4892 VI/1994 (HAS 34009); Arroio Sampainho, estação 2, VI/1994, lâmina n. 4901 (HAS 34016).

Subordem Naviculineae Hendey 1937

Família Naviculaceae Kütz. 1844

Navicula J.B.M Bory de St.-Vincent 1822

19. Navicula subminuscula Mang., Ver. Algol., 12: 139, est. 2, f. 39. 1941.

Fig. 20

Valvas elíptica-lanceoladas, com extremidades levemente cuneadas. Rafe linear reta. Área axial linear, área central indiferenciada. Comprimento $9,6 \mu \mathrm{m}$; largura $4,2-4,8 \mu \mathrm{m}$; estrias transapicais finas, levemente radiadas, $23 \mathrm{em} 10 \mu \mathrm{m}$. Material examinado: BRASIL. Rio Grande do Sul: Mato Leitão, Arroio Sampaio, estação 1, VI/1994, lâmina n. 4892 (HAS 34009); Arroio Sampaio, estação 1, XII/1994 lâmina n. 4896 (HAS 34013).

20. Navicula tenelloides Hust., Arch. Hydrobiol. Suppl. 15(2): 269, est. 19, f. 13. 1937.

Fig. 21
Valvas linear-lanceoladas com extremidades arredondadas. Rafe linear reta. Área axial linear estreita, área central irregular. Comprimento $18,6 \mu \mathrm{m}$; largura $4 \mu \mathrm{m}$; estrias transapicais finas, radiadas na região central e convergentes em direção às extremidades, $18 \mathrm{em} 10 \mu \mathrm{m}$.

Material examinado: BRASIL. Rio Grande do Sul: Mato Leitão, Arroio Sampaio, estação 1, XII/1994, lâmina n. 4896 (HAS 34013).

Comentários: primeira citação para o estado do Rio Grande do Sul.

Ordem Bacillariales Hendey 1937

Família Bacillariaceae Ehr. 1831

Tryblionella W. Smith 1853

21. Tryblionella victoriae Grun., Die Österreichischen Diatomaceen. 12: 553, est. 12, f. 34 a/b. 1862. Nitzschia tryblionella Hantz. var. victoriae (Grun.) Grun. in Cleve et Möller, Diatoms (Exsiccata) 211. 1878 .

Fig. 22

Valvas elípticas com dois planos distintos no eixo transapical. Extremidades lemente cuneadas. Rafe localizada ao longo da margem da valva. Comprimento $27-51 \mu \mathrm{m}$; largura 12,6$20 \mu \mathrm{m}$; estrias transapicais grosseiras, $7 \mathrm{em}$ $10 \mu \mathrm{m}$; fíbulas grandes, 6-7 em 10 $\mu \mathrm{m}$.

Material examinado: BRASIL. Rio Grande do Sul: Mato Leitão, Arroio Sampaio, estação 1, XII/1994, lâminas n. 4895 - 4896 (HAS 34013).

\section{Agradecimentos}

Á Fundação de Amparo à Pesquisa no Rio Grande do Sul (FAPERGS) pela concessão de Bolsa recém-mestre (Processo n. 97/60014-3) à primeira autora e ao Conselho Nacional de Desenvolvimento Científico e Tecnológico (CNPq) pela concessão de Bolsa Produtividade em Pesquisa (Processo n. 302050/83-3) à segunda autora. Ao auxiliar técnico Gilberto Machado, da Fundação Zoobotânica do Rio Grande do Sul, pela confecção das lâminas permanentes. 


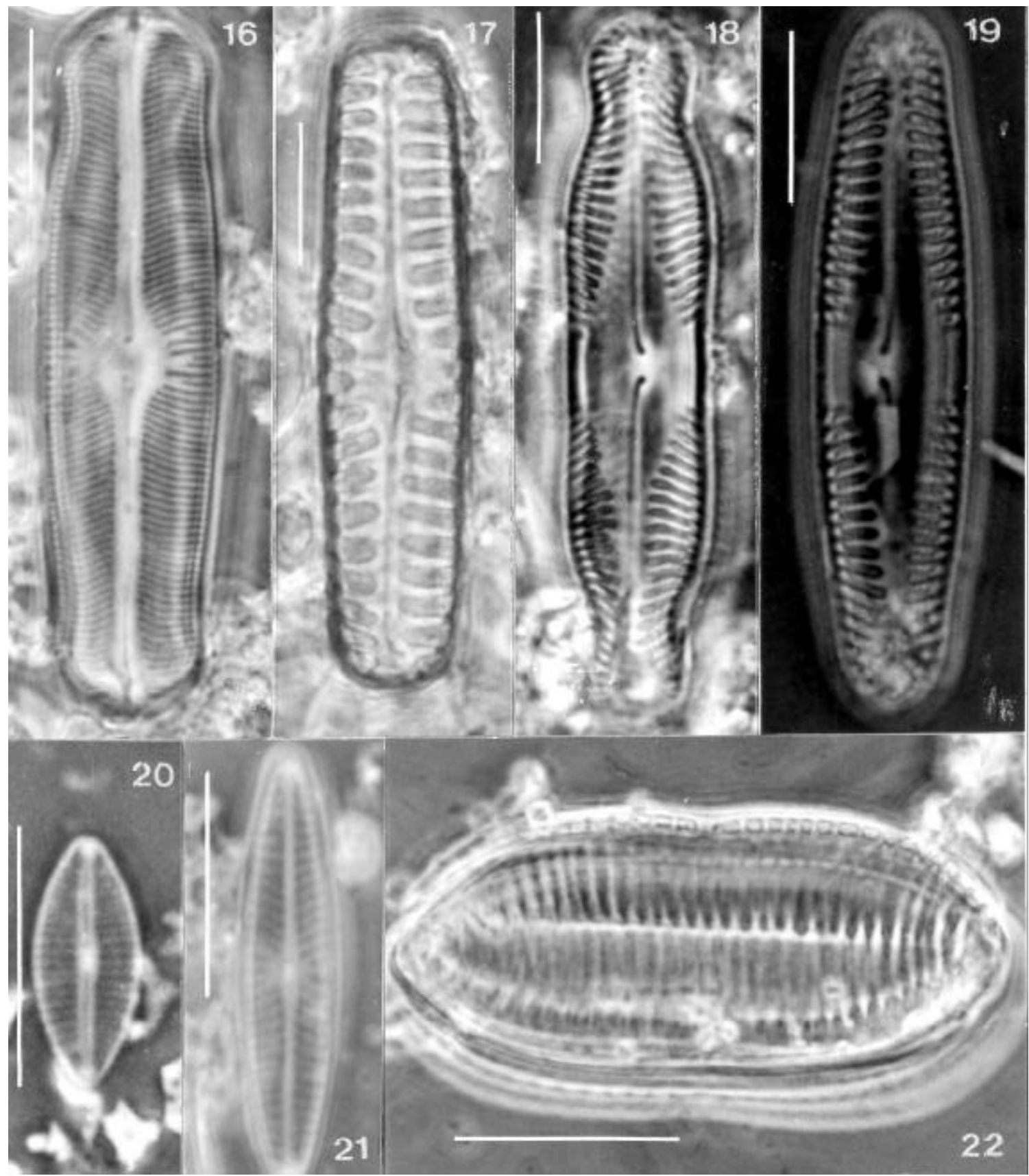

Figuras 16-22. 16. Sellaphora pupula; 17; Pinnularia borealis var. rectangularis; 18. Pinnularia mesolepta; 19. Pinnularia microstauron; 20. Navicula subminuscula; 21. Navicula tenelloides; 22. Tryblionella victoriae.

\section{Referências Bibliográficas}

Camburn, K.E., Lowe, R.L., Stoneburner, D.L. 1978. The Haptobenthic Diatom Flora of Long Branch Creek, South Carolina. Nova Hedwigia 30: 149-279.
Cleve-Euler, A. 1953. Die Diatomeen von Schweeden und Finland. Bihang K. Svenska Vetenskapsakademiens Handlingar 2 (1): 1-163.

Cox, E.J. 1987. Placoneis Mereschkowsky: the re-evaluation of a diatom genus originally characterized by its 
chloroplast type. Diatom Research 2(2):145-157.

Germain, H. 1981. Flore des Diatomées. Societé Nouvelle des Éditions Boubée, Paris.

Gough, S.B. \& Woelkerling, W.J. 1976. On the removal and quantification of algal aufwuchs from macrophyte hosts. Hydrobiologia 48 (3): 203-207.

Hartley, B. (Org.) 1996. An Atlas of British Diatoms. Biopress, Bristol.

Hohn, M.H. \& Hellerman, J. 1963. The taxonomy and structure of diatom populations from three eastern North American rivers using three sampling methods. Transactions of the American Microscopical Society 1 ( 82): 250-329.

Hürlimann, J. \& Straub, F. 1991. Morphologische und ökologishe charakterisierung von sippen um den Fragilaria capucina - komplex sensu Lange-Bertalot, 1980. Diatom Research 6 (1): 21-47.

Hustedt, 1930. Die Süsswasser-flora mitteleuropas. Heft 10: Bacillariophyta (Diatomeae). Jena.

Hustedt, F. 1937 - 1938. Systematische und ökologische untersuchungen über die diatomeen-flora von Java, Bali und Sumatra. Archiv für Hydrobilogie 15( 2):187-295.

Hustedt, F. 1961. Die Kieselalgen. L. Rabenhorst's Kryptogamen-Flora von Deutschland, Österreich und der Schweiz 7 (3): 1-160.

Jahn, R. 1986. A study of Gomphonema augur Ehrenberg: the structure of the frustule and is variability in clones e populations. Pp. 191-204. In: Ricard, M. (Ed.) Proceedings of the Eighth International Diatom Symposium, Koenigstein.

Krammer, K. \& Lange-Bertalot, H. 1986. Bacillariophyceae, 1: Naviculaceae. Pp 1-876. In: Ettl, H., Gerloff, J., Heynig, H. \& Mollenhauer, D. (Eds.) Die Süsswasserflora von Mitteleuropa. G. Fischer, Stuttgart.

Krammer, K. \& Lange-Bertalot, H. 1991a. Bacillariophyceae, 3: Centrales, Fragilariaceae, Eunotiaceae. Pp. 1-576. In: ETTL, H., GERLOFF, J., HEYNIG, H. \& MOLLENHAUER, D. (eds.) Die Süsswasserflora von Mitteleuropa. G. Fischer, . Stuttgart.

Krammer, K. \& Lange-Bertalot, H. 1991b. Bacillariophyceae, 4: Achnanthaceae. Pp. 1-437. In: ETTL,
H., GERLOFF, J., HEYNIG, H. \& MOLLENHAUER, D. (eds.) Die Süsswasserflora von Mitteleuropa. G. Fischer, Stuttgart.

Müller-Melchers, F. C. \& Ferrando, H. J. 1956. Técnica para el estudio de las diatomeas. Boletim do Instituto de Oceanografia 7 (1-2):151-60.

Oliveira, M.A. 1996. Ecologia do perifíton de substrato artificial em cursos d'água do trecho médio do Arroio Sampaio, Mato Leitão, RS, Brasil. Dissertação de Mestrado. Universidade Federal do Rio Grande do Sul, Porto Alegre.

Oliveira, M. A., Schwarzbold, A. 1998. Influência de fatores abióticos sobre a estrutura da comunidade perifítica de limnotopos da Bacia do Arroio Sampaio, Mato Leitão, Rio Grande do Sul. Iheringia, Sér. Botânica 51(1): 39-63.

Oliveira, M. A., Torgan, L.C., Lobo, E.A. \& Schwarzbold, A. 2001. Associação de espécies de diatomáceas perifíticas de substrato artificial em ambientes lóticos da Bacia do Arroio Sampaio, RS, Brasil: relação com variáveis abióticas. Revista Brasileira de Biologia 61 (4): 523-540.

Patrick, R. \& Reimer, C.W. 1966. The diatoms of the United States, exclusive of Alaska and Hawaii. Livingstgon Company, Philadelphia.

Patrick, R. \& Reimer, C.W. 1975. The diatoms of the United States, exclusive of Alaska and Hawaii. Livingstgon Company, Philadelphia.

Rivera, P. R. 1974. Diatomeas de agua dulce de Concepcion y alrededores (Chile). Gayana (28): 3-134.

Round, F.E., Crawford, R.M. \& Mann, D.G. 1990. The Diatoms - biology and morphology ot the genera. Cambridge University Press, Cambridge.

Schoeman, F.R. \& Archibald, R.E.M. 1978. The Diatom Flora of Southern Africa n. 4. Council for Scientific and Industrial Research, Pretoria.

Schoeman, F.R. \& Archibald, R.E.M. 1979. The Diatom Flora of Southern Africa n. 5. Council for Scientific and Industrial Research, Pretoria.

Van Heurck, H. 1885. Synopsis des Diatomées de Belgique. Linnaeus Press, Amsterdam.

Williams, D.M. \& Round, F.E. 1987. Revision of the genus Fragilaria. Diatom Research 2 (2): 267-288. 